\title{
Parameter estimation with mixed quantum states
}

\author{
Daniel Braun ${ }^{1,2}$ \\ 1 Université de Toulouse, UPS, Laboratoire de Physique \\ Théorique (IRSAMC), F-31062 Toulouse, France and \\ 2 CNRS, LPT (IRSAMC), F-31062 Toulouse, France
}

\begin{abstract}
We consider quantum enhanced measurements with initially mixed states. We show very generally that for any linear propagation of the initial state that depends smoothly on the parameter to be estimated, the sensitivity is bound by the maximal sensitivity that can be achieved for any of the pure states from which the initial density matrix is mixed. This provides a very general proof that purely classical correlations cannot improve the sensitivity of parameter estimation schemes in quantum enhanced measurement schemes.

PACS numbers:
\end{abstract}


Quantum enhanced measurements aim at exploiting quantum mechanical effects for improving the sensitivity of measurements of classical parameters of a system. The most-well known examples are the improvement of the measurement of phase shifts in an interferometer by using squeezed light [1], or the idea of using NOON states for super-resolution [2]. In 1994 Braunstein and Caves developed a general theory of quantum enhanced measurements, based on a generalization to the quantum world of the celebrated Cramér-Rao bound in classical parameter estimation theory [3, 4]. Indeed, even classically the problem of the optimal strategy of determining the value of a parameter $x$ that parametrizes a probability distribution, and the best achievable sensitivity, are of fundamental importance in many branches of science. In quantum mechanics, one would like to get the best possible estimate of a variable $x$ that parametrizes a general quantum state $\rho(x)$, based on arbitrary measurements, repeated eventually $N$ times in an always identically prepared state. In the above example of an interferometer, $\rho(x)$ would be the state at the output of the interferometer, and $x$ the phase shift we want to measure. The latter might itself depend on a number of interesting parameters, such as the length of an arm of the interferometer in the case of a Mach-Zehnder interferometer, the absorption index of a probe brought into one of the arms, or the magnetic field acting on a spin in the case of Ramsey interferometry.

Braunstein and Caves showed [3] that the best achievable sensitivity for measuring $x$ in the state $\rho(x)$ is given by

$$
\left\langle\delta x^{2}\right\rangle_{\rho(x)}^{1 / 2} \geq\left.\frac{1}{\sqrt{N}\left(\frac{d s^{2}}{d x^{2}}\right)^{1 / 2}} \equiv \delta x_{\min }\right|_{\rho(x)} .
$$

Here, $d s^{2}=4 d_{\text {Bures }}^{2}(\rho(x), \rho(x)+d \rho)$ is given by the so-called Bures distance, which will be defined precisely below, and $d \rho$ denotes the infinitesimal change of $\rho(x)$ when $x$ varies about its initial value for which we want to know the best achievable sensitivity. It is important to note that the bound (11) can be saturated in the limit of large $N$.

Based on (11), a number of important results were obtained. Notably, it was shown that for an initial product state $|\psi\rangle=\otimes_{i=1}^{K}|\phi\rangle_{i}$ of $K$ sub-systems (i.e. $K$ "quantum resources"), unitary evolution with a hamiltonian $H(x)=x \sum_{i=1}^{K} h_{i}$, and arbitrary final projective mea- 
surements, the best achievable sensitivity is

$$
\left.\delta x_{\min }\right|_{|\psi(x)\rangle\langle\psi(x)|}=\frac{1}{\sqrt{N K}(\Lambda-\lambda)},
$$

where $|\psi(x)\rangle=\exp (-i H(x))|\psi\rangle$, and $\Lambda$ and $\lambda$ are the largest and smallest eigenvalue of $h_{i}$, respectively (the $h_{i}$ are taken identical for all subsystems). On the other hand, if the initial state is fully entangled over all $K$ subsystems, the same unitary evolution allows to achieve a sensitivity

$$
\left.\delta x_{\min }\right|_{|\psi(x)\rangle\langle\psi(x)|}=\frac{1}{\sqrt{N} K(\Lambda-\lambda)},
$$

i.e. an improvement by a factor $1 / \sqrt{K}[\underline{5}]$.

The development of quantum enhanced measurements has focused so far on pure initial states. These are either entangled or product states. The decisive degree of freedom is then the number of subsystems over which entanglement extends. In the case of mixed states, there is more freedom, as classical correlations and entanglement might coexist. The question thus arises what can be achieved with general initial mixed states, and in particular whether classical correlations may improve quantum enhanced measurements. In this Brief Report we prove a no-go theorem, which essentially says that with a mixed state one cannot achieve better sensitivity than with any of the pure states from which it is mixed. In order to prove this result, we first show a little Lemma.

Lemma. The squared Bures distance is joint-convex, i.e. for two arbitrary states $\rho, \sigma$ we have

$$
d s_{\text {Bures }}^{2}\left(a \rho_{1}+(1-a) \rho_{2}, a \sigma_{1}+(1-a) \sigma_{2}\right) \leq a d s_{\text {Bures }}^{2}\left(\rho_{1}, \sigma_{1}\right)+(1-a) d s_{\text {Bures }}^{2}\left(\rho_{2}, \sigma_{2}\right) .
$$

Proof. The squared Bures distance is defined as

$$
d s_{\text {Bures }}^{2}(\rho, \sigma) \equiv 2(1-\sqrt{F(\rho, \sigma)})
$$

where the fidelity $F(\rho, \sigma)$ is given by $F(\rho, \sigma)=\left\|\rho^{1 / 2} \sigma^{1 / 2}\right\|_{1}^{2}$, and $\|A\|_{1} \equiv \operatorname{tr} \sqrt{A A^{\dagger}}$ denotes the trace norm [6]. It is known that $\sqrt{F(\rho, \sigma)}$ is joint-concave [6], i.e. we have

$$
\sqrt{F\left(a \rho_{1}+(1-a) \rho_{2}, a \sigma_{1}+(1-a) \sigma_{2}\right)} \geq a \sqrt{F\left(\rho_{1}, \sigma_{1}\right)}+(1-a) \sqrt{F\left(\rho_{2}, \sigma_{2}\right)} .
$$


This immediately gives the joint-convexity of the squared Bures distance,

$$
\begin{aligned}
d s_{\text {Bures }}^{2}\left(a \rho_{1}+(1-a) \rho_{2}, a \sigma_{1}+(1-a) \sigma_{2}\right) & =2\left(1-\sqrt{F\left(a \rho_{1}+(1-a) \rho_{2}, a \sigma_{1}+(1-a) \sigma_{2}\right)}\right) \\
& \leq 2\left(1-\left(a \sqrt{F\left(\rho_{1}, \sigma_{1}\right)}+(1-a) \sqrt{F\left(\rho_{2}, \sigma_{2}\right)}\right)\right) \\
& \leq a 2\left(1-\sqrt{F\left(\rho_{1}, \sigma_{1}\right)}\right)+(1-a) 2\left(1-\sqrt{F\left(\rho_{2}, \sigma_{2}\right)}\right) \\
& \leq a d_{\text {Bures }}^{2}\left(\rho_{1}, \sigma_{1}\right)+(1-a) d_{\text {Bures }}^{2}\left(\rho_{2}, \sigma_{2}\right)
\end{aligned}
$$

which proves the Lemma.

With this Lemma, it is straightforward to show the following theorem:

Theorem. Let $\rho_{0}=\sum_{i} p_{i}\left|\psi_{i}\right\rangle\left\langle\psi_{i}\right|$ be an arbitrary mixed state $\left(0 \leq p_{i} \leq 1, \sum_{i} p_{i}=1\right)$, and $\rho(x)=\mathcal{L}_{x}\left[\rho_{0}\right]$ the state generated from $\rho_{0}$ by an arbitrary linear map $\mathcal{L}_{x}$ which depends smoothly on the parameter $x$. Then the sensitivity for measuring $x$ in the state $\rho(x)$ is bounded by the best achievable sensitivity in the state obtained by propagating any of the pure states $\left|\psi_{i}\right\rangle$ with the same map, i.e.

$$
\left.\delta x_{\min }\right|_{\rho(x)} \geq\left.\delta x_{\min }\right|_{\mathcal{L}_{x}\left[\left|\psi_{0}\right\rangle\left\langle\psi_{0}\right|\right]}
$$

where $\left|\psi_{0}\right\rangle$ is the pure state in the set of all $\left\{\left|\psi_{i}\right\rangle\right\}$ which leads to the best sensitivity, i.e. $\left.\min _{\left\{\left|\psi_{i}\right\rangle\right\}} \delta x_{\min }\right|_{\mathcal{L}_{x}\left[\left|\psi_{i}\right\rangle\left\langle\psi_{i}\right|\right]}=\left.\delta x_{\min }\right|_{\mathcal{L}_{x}\left[\left|\psi_{0}\right\rangle\left\langle\psi_{0}\right|\right]}$.

Proof. Due to the linearity of $\mathcal{L}_{x}[\rho]$, we have

$$
\rho(x)=\mathcal{L}_{x}\left[\rho_{0}\right]=\sum_{i} p_{i} \mathcal{L}_{x}\left[\left|\psi_{i}\right\rangle\left\langle\psi_{i}\right|\right]
$$

and thus, for smooth $x$-dependence of $\mathcal{L}_{x}$,

$$
d \rho=\frac{d \rho}{d x} d x=\sum_{i} p_{i} d x \mathcal{L}_{x}^{\prime}\left[\left|\psi_{i}\right\rangle\left\langle\psi_{i}\right|\right],
$$

where $\mathcal{L}_{x}^{\prime}$ denotes the derivative of $\mathcal{L}_{x}$ with respect to $x$. Therefore,

$$
\begin{aligned}
d_{\text {Bures }}^{2}(\rho(x), \rho(x)+d \rho) & =d_{\text {Bures }}^{2}\left(\sum_{i} p_{i} \mathcal{L}_{x}\left[\left|\psi_{i}\right\rangle\left\langle\psi_{i}\right|\right], \sum_{i} p_{i}\left(\mathcal{L}_{x}\left[\left|\psi_{i}\right\rangle\left\langle\psi_{i}\right|\right]+d x \mathcal{L}_{x}^{\prime}\left[\left|\psi_{i}\right\rangle\left\langle\psi_{i}\right|\right]\right)\right) \\
& \leq \sum_{i} p_{i} d_{\text {Bures }}^{2}\left(\mathcal{L}_{x}\left[\left|\psi_{i}\right\rangle\left\langle\psi_{i}\right|\right], \mathcal{L}_{x}\left[\left|\psi_{i}\right\rangle\left\langle\psi_{i}\right|\right]+d x \mathcal{L}_{x}^{\prime}\left[\left|\psi_{i}\right\rangle\left\langle\psi_{i}\right|\right]\right) \\
& \leq d_{\text {Bures }}^{2}\left(\mathcal{L}_{x}\left[\left|\psi_{0}\right\rangle\left\langle\psi_{0}\right|\right], \mathcal{L}_{x}\left[\left|\psi_{0}\right\rangle\left\langle\psi_{0}\right|\right]+d x \mathcal{L}_{x}^{\prime}\left[\left|\psi_{0}\right\rangle\left\langle\psi_{0}\right|\right]\right),
\end{aligned}
$$

where we have designated $\left|\psi_{0}\right\rangle$ to be the state which maximizes $d_{\text {Bures }}^{2}\left(\mathcal{L}_{x}\left[\left|\psi_{i}\right\rangle\left\langle\psi_{i}\right|\right], \mathcal{L}_{x}\left[\left|\psi_{i}\right\rangle\left\langle\psi_{i}\right|\right]+d x \mathcal{L}_{x}^{\prime}\left[\left|\psi_{i}\right\rangle\left\langle\psi_{i}\right|\right]\right)$ over all $\left|\psi_{i}\right\rangle$, and thus minimizes 
$\left.\delta x_{\min }\right|_{\mathcal{L}_{x}\left[\left|\psi_{i}\right\rangle\left\langle\psi_{i}\right|\right]}$. Plugging this upper bound on $d_{\text {Bures }}^{2}$ back into (11) and taking the square root immediately shows the theorem.

Since there are many ways of mixing a given density matrix from pure states, the usefulness of the theorem for obtaining bounds on the best sensitivity achievable may depend on the chosen decomposition of $\rho_{0}$. For example, one may start with a pure state that is entangled over all subsystems, and admix to it a separable state (see e.g.[7]). Then the lower bound on $\delta x_{\min }$ remains given by the entangled state. However, for a sufficiently large admixture of the separable state, the initially entangled state might become at least partially separable, and a new decomposition of $\rho(x)$ in terms of pure states can be found, which raises the lower bound on $\delta x_{\min }$. The most straightforward and useful application of the theorem arises in the case of an initially fully separable state $\rho_{0}$. In this case the theorem immediately shows that one cannot achieve a better sensitivity than with the initial pure product states from which it is mixed, i.e. in particular a scaling as $1 / \sqrt{K}$ with the number of quantum resources $K$ for the example mentioned in the introduction. This generalizes the findings of [5] for the CC and CQ scenarios (classical initial state, taken as a pure product state in [5], and factorizing or entangling measurement) to mixed separable states.

Very recently a preprint appeared which came to the same conclusion using a different method, and in a less general setting [8].

[1] C. M. Caves, Phys. Rev. D 23, 1693 (1981).

[2] A. N. Boto, P. Kok, D. S. Abrams, S. L. Braunstein, C. P. Williams, and J. P. Dowling, Physical Review Letters 85, 2733 (2000).

[3] S. L. Braunstein and C. M. Caves, Phys. Rev. Lett. 72, 3439 (1994).

[4] H. Cramér, Mathematical Methods of Statistics (Princeton University Press, Princeton, NJ, 1946).

[5] V. Giovannetti, S. Lloyd, and L. Maccone, Phys. Rev. Lett. 96, 010401 (2006).

[6] J. A. Miszczak, Z. Puchała, P. Horodecki, A. Uhlmann, and K.Życzkowski, Quantum Information and Computation 9, 0103 (2009). 
[7] R. F. Werner, Phys. Rev. A 40, 4277 (1989).

[8] G. Goldstein, M. D. Lukin, and P. Cappellaro, Quantum limits on parameter estimation, arXiv:1001.4804v1. 